\title{
La gravedad: fuerza, geometría e ilusión
}

\author{
Jorge Oswaldo Calderón Aguirre \\ Universidad Central del Ecuador \\ jocalderon@uce.edu.ec
}

Recibido: 01 de septiembre / Aprobado: 30 de noviembre 2017

\section{Resumen}

La gravedad ha sido, es y será posiblemente por muchísimo tiempo un completo misterio para la humanidad y la ciencia, la actuación de la gravedad en el universo es omnipotente y omnipresente, por lo que desenmarañar su funcionamiento físico y matemático siempre ha sido un desafío para las mentes más brillantes de la humanidad; para resumir el esfuerzo efectuado por las distintas generaciones, se realiza un repaso sucinto de los pensamientos de grandes hombres como Aristóteles pasando por Newton y arribando a Einstein. Se menciona también las teorías que explican de mejor manera el accionar de la gravedad en el micro y macro cosmos (la mecánica cuántica y la relatividad general). Constituyendo la gravedad una de las fuerzas fundamentales del universo, se menciona asimismo a las otras, es decir la electro-magnética, la fuerte y la débil.

Palabras clave: gravedad, fuerzas fundamentales, espacio-tiempo, cuántica, gravitón.

\section{Abstract}

Gravity has been, is and will be for a very long time a complete mystery to mankind and science, the action of gravity in the Universe is omnipotent and omnipresent, 
so unraveling its physical and mathematical functioning has always been a challenge for the most brilliant minds of mankind; to sum up the effort made by the different generations, a brief review is made of the thoughts of great men like Aristotle passing through Newton and arriving to Einstein. It also mentions theories that best explain the action of gravity in the micro and macro cosmos (quantum mechanics and general relativity). Gravity being one of the fundamental forces of the Universe, it is also in relation to the others, to say the electro-magnetic, the strong and the weak.

Keywords: gravity, fundamental forces, space-time, quantum, graviton. 


\section{Introducción}

L

a gravedad es una de las fuerzas fundamentales del universo, la que mantiene unido todos los cuerpos formados por materia; en nuestro planeta Tierra nos galaxias, en general la gravedad es lo que da unidad y cohesión al cosmos. Con esta sencilla y ligera introducción, y más aún con un limitado conocimiento de la física teórica y experimental, y mucho menos de la astrofísica; me atrevería a señalar que la gravedad es el "pegamento" del universo. Les invito a continuación a que ampliemos nuestros conocimientos un poco más, reconociendo las características básicas que definen la gravedad.

\section{Materiales y métodos}

El método de investigación utilizado en el presente trabajo es eminentemente cualitativo, desarrollado principalmente mediante la recopilación de información de texto y video (documentales), que se encuentran en la web; la información allí obtenida fue sintetizada de forma cronológica y luego analizada de forma interpretativa y subjetiva. Con ello se logra alcanzar el objetivo principal de la presente investigación, que es brindar una descripción histórica de las diferentes teorías de la gravedad y sus autores. Esta búsqueda es ideal como fase inicial de un gran proyecto de investigación que brinde una descripción completa y detallada del accionar de la gravedad en el universo.

\section{Discusión}

Es muy importante señalar, que todo cuerpo conformado por materia tiene gravedad, solo que la misma es notable, en cuerpos de tamaños enormes como los planetas y el Sol.

La fuerza gravitacional es diferente en cada planeta, esto va a depender de la masa del mismo. Para un entendimiento más claro tenemos que diferenciar entre lo que definimos como "masa" y lo que conocemos como "peso". Son conceptos muy diferentes: la masa (cantidad de materia que tiene un cuerpo) da igual si la medimos aquí o en cualquier otro planeta, en cambio el peso es una fuerza que sí depende de la masa del planeta donde lo midamos (un kilogramo, que en la Tierra pesa un kilopondio, en Júpiter pesaría dos kilopondios y medio). [1]

Mencionemos las ideas históricas más relevantes de filósofos, astrónomos y científicos (físicos - matemáticos) que se "atrevieron" a desenmarañar el funcionamiento de la gravedad: En el siglo IV a.C., Aristóteles motivado por su teoría causa-efecto, basada únicamente en la observación y la abstracción (no tomaba en cuenta la experimentación), pensó que una fuerza invisible nos atraía hacia el centro del universo, para ese entonces la Tierra era el centro del universo y todo gira a su alrededor (teoría 
geocéntrica); este pensamiento tenía un gran error, el de creer que existía una gravedad distinta según la masa del cuerpo, a decir de esto la gravedad "no tiene un valor constante"; esta interpretación de dicho fenómeno duró aproximadamente veinte siglos. [2] Llegado el siglo XVI d.C. Nicolás Copérnico provocó una verdadera revolución en lo poco que se conocía de astronomía, al manifestar que el Sol era el centro del universo, la Tierra es la que gira alrededor del Sol; pensamiento (teoría heliocéntrica) que permitió corregir uno de los errores cometidos por Aristóteles. Entre los siglos XVI y XVII d.C. Galileo Galilei pionero del método científico experimental, investigó la caída de los cuerpos, mediante experimentos en planos inclinados y en la torre de Pisa, llegando a la conclusión de que la gravedad era una "fuerza constante" en todos los cuerpos, enmendando otro error del pensamiento de Aristóteles; también logró perfeccionar el telescopio, que le permitió realizar observaciones que lo convencieron de la certeza de la teoría heliocéntrica de Copérnico. [3] En esas mismas épocas un pastor protestante Johannes Kepler, firme seguidor de la teoría de Copérnico, describió al fenómeno que se trata, como un complejo sistema creado por Dios para conseguir que los planetas se mantengan girando (leyes de Kepler); la teoría más aceptada para dar respuestas a las incógnitas generadas por esta afirmación, partía de la existencia de dos fuerzas de gravedad, la una que actúa en los objetos sobre la superficie de la Tierra y la otra que parece ser una "fuerza celestial" que logra que los planetas giren. Luego pasemos a mencionar las ideas de Isaac Newton (1687) y Albert Einstein (1915) sobre cómo actúa la fuerza de la gravedad (interacción gravitatoria). A quienes debemos las dos mejores teorías de la gravitación, son probablemente los más grandes físicos de la historia de la humanidad. [4]

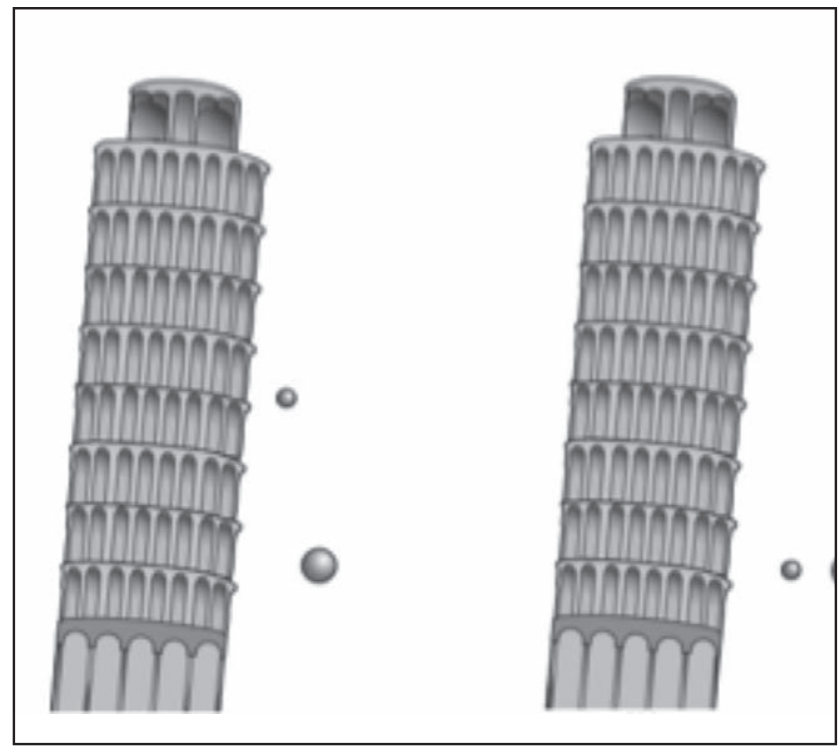

Figura 1. Experimento de Galileo Galilei. 
Newton observó el hecho de que los cuerpos "pesaban" en la Tierra (estos flotan en el espacio interestelar) y que los cuerpos celestes giren alrededor de otros cuerpos celestes (la Luna alrededor de la Tierra, la Tierra y los otros planetas alrededor del Sol, y así todos los cuerpos celestes) y se "imaginó" que existía una fuerza universal que producía que los cuerpos se atrajeran entre sí, además que actúa en todos los lugares del cosmos. Esta interacción se manifiesta tanto en la atracción de un cuerpo producida por la Tierra (su peso), como por la atracción entre todos los cuerpos celestes del universo, la misma que les hace girar unos alrededor de otros. Newton llamó a esta maravilla "ley de la gravitación universal" ("Dos cuerpos cualesquiera de masas $\mathrm{m} 1$ y $\mathrm{m} 2$, separados por una distancia " $\mathrm{r}$ ", se atraen con una fuerza cuya magnitud es directamente proporcional al producto de las masas e inversamente proporcional al cuadrado de la distancia que los separa"). Según Newton, la gravedad es una fuerza instantánea, cualquier cuerpo advertiría inmediatamente la presencia de otro cuerpo y soportaría su atracción, y actuaría a distancia, sin que haya contacto entre los cuerpos. La pregunta que surge de inmediato es ¿por qué existe esa fuerza?, Newton no lo supo explicar satisfactoriamente, la imputó a la Divinidad (Divinitas). Sin embargo, gracias a su teoría, físicos, astrónomos e ingenieros han logrado comprender con altísima precisión las órbitas de los planetas, la dinámica de las galaxias, las mareas causadas por la Luna y el Sol, y también nos ha sido posible construir naves espaciales que viajan alrededor de nuestro planeta y por los confines del sistema solar.

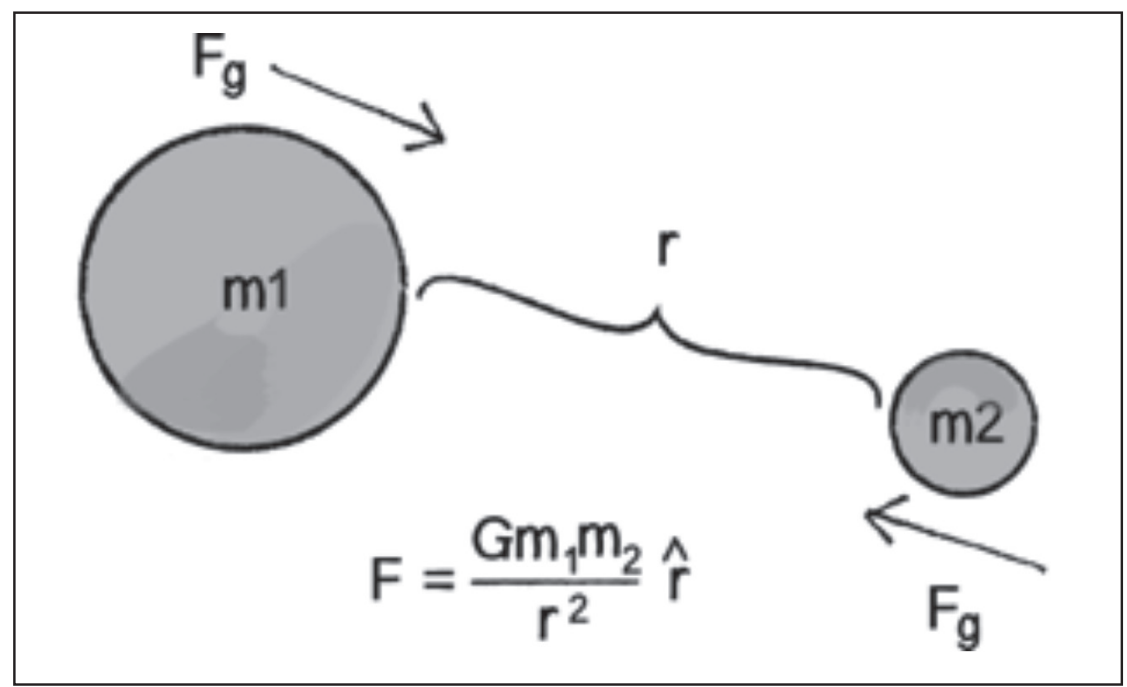

Figura 2. Ley de la gravitación universal.

Albert Einstein en 1915, propuso que la gravedad era una "ilusión" y no una fuerza de atracción. Según él "La gravedad es un efecto de la geometría. La Tierra desfigura el espacio-tiempo de nuestro contexto, de tal manera que el propio espacio nos empuja 
hacia el suelo"; esta interpretación del fenómeno denominado gravedad pertenece a la "teoría de la relatividad general" (perfectamente aplicable al mundo macroscópico o el de los objetos grandes). Entender la relatividad general de Einstein no es fácil. En el universo la gravedad acelera a todos los cuerpos, poniéndolos en movimiento; dada esta realidad, podemos garantizar que nada está en reposo, todo el universo se mueve y con aceleración. Además según el mismo Einstein y su formidable "teoría de la relatividad especial", la velocidad de la luz es un límite cósmico, que nada en el universo puede superar (según Newton la gravedad tiene acción instantánea). Recapitulando, la genial idea de Einstein fue suponer que la gravedad (que está en todos los costados y en todo instante en el universo) está íntimamente unida al espacio y al tiempo. Propuso que el nexo de unión era la geometría: lo que ocurre, es que, en presencia de una masa, el espacio-tiempo se "deforma", de modo que cualquier otra masa se percata de ese espacio deformado, y se ve obligado a seguir trayectorias diferentes a cuando estaba el espacio sin deformar (sin ninguna masa). La deformación del espacio significa que éste adquiere una geometría diferente a la que estamos acostumbrados, llamado espacio plano o euclidiano. A saber, en un espacio no euclidiano ocurren cosas muy diferentes, como por ejemplo que la línea más corta entre dos puntos sea una curva y no una recta, o que dos paralelas se corten en un punto; estas ideas abstractas las podemos visualizar, en un simple globo terráqueo. Para llegar a construir su "nueva" teoría de la gravedad, Einstein en su línea de razonamiento partió de dos sucesos: en que nada puede propagarse más rápido que la luz (relatividad especial), y en el planteamiento de un fenómeno hipotético de la desaparición instantánea del Sol (un experimento mental), surgiendo de la reflexión de estos hechos, un contrasentido con respecto a la velocidad de transmisión de la información originadas por un mismo acontecimiento (la desaparición del Sol); la luz viaja a la velocidad ya conocida $(300.000 \mathrm{~km} / \mathrm{s})$ y la acción gravitacional a una velocidad infinita, ante este dilema surgió su ingeniosa salida, proponer la "teoría de la relatividad general”, descripción más aceptada de la gravedad. [5]

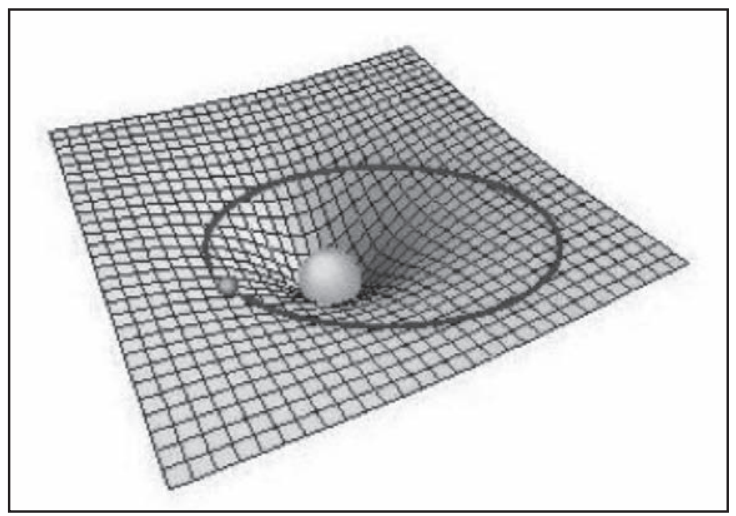

Figura 3. Deformación del espacio-tiempo. 
La "mecánica cuántica" (parte de la física que estudia el movimiento de las partículas muy pequeñas) y la "relatividad general" son teorías que describen con gran exactitud la realidad del micro y macro cosmos, sí una es correcta lo otra no lo sería, surge un reto para los físicos teóricos, el proponer un nuevo modelo de teoría general. [6] Actualmente se cree que la mecánica cuántica es la más acertada y se trabaja en proponer una "teoría cuántica de la gravedad". La gravedad con su alcance infinito y con su capacidad de mantener grandes estructuras (planetas, galaxias), es la más débil de las interacciones fundamentales y no puede ser aplicada a las partículas. Del mismo modo en que el fotón es la partícula cuántica de la fuerza electromagnética, el gluon es el átomo de la fuerza nuclear fuerte y el conocido como "gravitón" es una hipotética partícula cuántica de la fuerza gravitacional.

Siendo la gravedad una de las interacciones fundamentales de la naturaleza, cabe un ligero repaso histórico por las demás. Ha sido muy entretenido para la humanidad, fundamentalmente para los físicos, determinar por cuántas fuerzas físicas estaba gobernado el mundo. En la antigüedad, Aristóteles consideró la existencia de "una" sola interacción (fuerza), que era la de la gravedad. Más adelante, en la misma época; se creía que "tres" eran esas fuerzas: la gravitatoria, la electricidad y el magnetismo. Luego se produce una primera unificación, gracias a los trabajos de Oersted, Faraday y particularmente Maxwell (1860); así que para ese entonces se pensó que solo había "dos" fuerzas: la gravitatoria y la electromagnética. [7] Durante el siglo $\mathrm{XX}$, un conocimiento casi inimaginable de la estructura microscópica de la materia, conduce a la identificación de nuevas interacciones, la interacción débil y la fuerte; por consiguiente ya eran "cuatro" las fuerzas que regían el mundo. Este relato no concluye ahí, en los sesenta del anterior siglo, Weinberg-Salam proponen una teoría que establece que la fuerza electromagnética y la fuerza débil son una sola, hecho ya demostrado experimentalmente, por lo que sin duda actualmente se afirma que existen "tres" fuerzas. Hoy en día los físicos vienen trabajando en la denominada "teoría de la gran unificación", de llegarse a comprobarla experimentalmente solo quedarían "dos" fuerzas (la gravitatoria y la electro-magneto-fuerte-débil). [8]

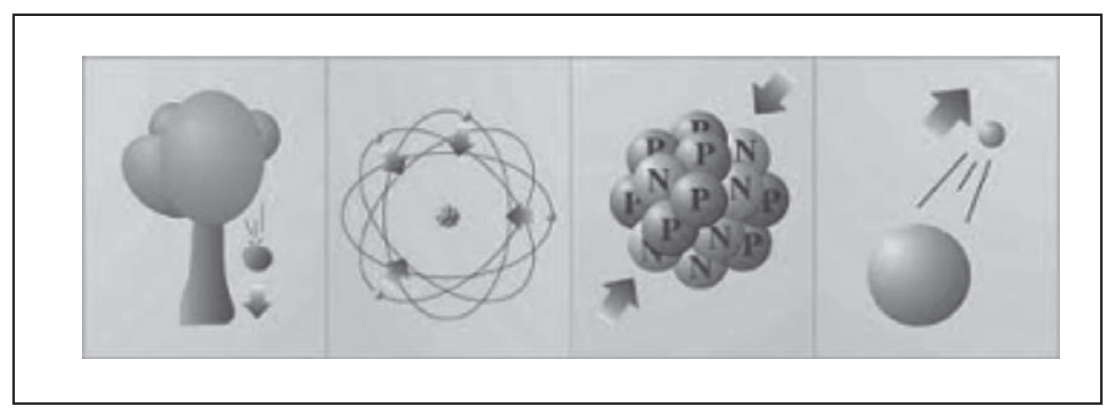

Figura 4. Fuerzas fundamentales de la naturaleza. 


\section{Conclusiones}

La gravedad es la fuerza más poderosa del universo, funciona tanto a gran escala como a escala infinitesimal.

La teoría de Newton carece de elementos que consideren la velocidad de la acción gravitacional a distancia, supone su actuación de forma instantánea (velocidad infinita); esto no es posible según Einstein (teoría de la relatividad general) puesto que la máxima velocidad de propagación de cualquier seńal es la velocidad de la luz.

Según la relatividad general, la presencia de una masa produce una curvatura del espacio que la rodea; mientras otro cuerpo que se encuentra a cierta distancia experimenta esa geometría, que lo empuja como si fuera una fuerza.

Finalmente puedo afirmar que, aún no tenemos una teoría final sobre la gravitación, es decir todavía no sabemos en realidad qué es la gravedad.

\section{Referencias}

[1] ¿Qué es la gravedad?, http://www.vix.com/es/btg/curiosidades/4429/que-es-lagravedad, septiembre 2017.

[2] En realidad, ¿qué es la gravedad?, https://spaceplace.nasa.gov/what-is-gravity/ sp/, septiembre 2017.

[3] Definición de gravedad, http://conceptodefinicion.de/gravedad/, septiembre 2017.

[4] ¿Qué es la gravedad?, https://espaciociencia.com/que-es-la-gravedad/, septiembre 2017.

[5] Gravitación-física avanzada, http://www.iac.es/cosmoeduca/gravedad/fisica/ fisica5.htm, agosto 2017.

[6] Interacciones fundamentales I: Gravedad, https://conexioncausal.wordpress. com/2010/05/05/interacciones-fundamentales-i-gravedad/, agosto 2017.

[7] Fuerza de gravedad, https://www.portaleducativo.net/quinto-basico/100/Fuerza-de-gravedad, agosto 2017.

[8] Las fuerzas fundamentales del universo, http://www.astromia.com/astronomia/fuerzasfundamentales.htm, agosto 2017.

[9] Documental la gravedad, https://www.youtube.com/watch?v=NZ2CtC07pmc, agosto 2017.

[10] La gravedad, fuerza más poderosa del universo, documental-2017, https:// www.youtube.com/watch?v=Lqz8kiRMajM, agosto 2017.

[11] La gravedad, https://www.youtube.com/watch?v=SZhzEpuTCbc, agosto 2017.

[12] La teoría de la relatividad de Einstein para todos los públicos, https://www. youtube.com/watch?v=heCBnyPn1YY, agosto 2017. 